\title{
Mass Spectrometry-Based Protein Biomarker Discovery and Measurement: Sensitivity is the Greatest Hurdle
}

\author{
Lance A. Liotta $\cdot$ Emanuel F. Petricoin
}

Published online: 21 April 2010

(C) Springer Science+Business Media, LLC 2010

One of the greatest clinical needs in cancer diagnostics is the accurate detection of early-stage tumors that have not yet metastasized. Treatment of such early lesions is expected to have a direct impact on long-term survival. In contrast, once dissemination and organ colonization has begun, the effectiveness of all conventional therapy is limited. By definition, such early-stage lesions are very small (less than $0.5 \mathrm{~cm}$ ) and often exist below the threshold of detection by sophisticated imaging such as MRI. Brown and Palmer [1] have estimated that a test for ovarian cancer would have to detect tumors smaller than $0.5 \mathrm{~cm}$ to achieve a $50 \%$ reduction in cancer mortality with an annual screen. Putative biomarkers elaborated into the circulation from a small pre-metastatic lesion will be diluted in the entire blood volume and can be subject to rapid clearance by a variety of mechanisms [2]. Thus, the expected concentration of a blood-borne diagnostic analyte derived from these crucially important, but tiny, clinical lesions will be much less than $1 \mathrm{ng} / \mathrm{ml}$ and will likely fall in the picograms-permilliliter range $[1,2]$.

Despite the rapid advancements in the application of mass spectrometry (MS) to biomarker discovery and quantitative measurement [3], serious physiologic challenges still remain that limit the sensitivity of MS. Consequently, the analytical sensitivity of MS for biomarker discovery is actually very low, particularly when applied to complex protein mixtures such as blood. Cancer-associated blood biomarkers exist within a sea of high-abundance proteins

L. A. Liotta $(\bowtie) \cdot$ E. F. Petricoin

Center for Applied Proteomics and Molecular Medicine,

George Mason University,

Manassas, VA 20110, USA

e-mail: 1liotta@gmu.edu such as albumin and immunoglobulins. Moreover, circulating biomarkers are subjected to degradation during transportation and storage, further reducing their concentration. In part because of these physiologic limitations, the vast majority of hundreds of clinical analytes measured routinely in the clinical chemistry laboratory today cannot be detected by current MS discovery approaches [4]. MS is simply not sensitive enough to detect the typical clinical analyte that falls in the range of $50 \mathrm{pg} / \mathrm{ml}$ to $5 \mathrm{ng} / \mathrm{ml}$. Established clinical immunoassays stand as tough competition because they are highly precise, reliable, and low in cost. In its current form, the routine sensitivity threshold MRM technology [3] is tens of nanograms per milliliter. The sensitivity of MRM needs to be improved 100 -fold in order to compete with clinical immunoassays. This is a sobering reality for proponents of MS who hope it will replace conventional immunoassays.

The majority of proteins cataloged by MS under the HUPO human plasma proteome project falls in the highabundance range [4]. Thus, a biomarker considered by MS to be negative in a sample may actually be present in the sample at a concentration below the level of MS sensitivity. A more sensitive technology such as protein array or ELISA may therefore detect a candidate biomarker in a control sample considered to be negative by MS. This should not be considered a false positive.

Fortunately, advances in nanotechnology, and affinity capture concentration, are providing a completely new approach to increase the sensitivity of MS for biomarker discovery [5-7]. Biomarker "harvesting" nanoparticles rapidly concentrate low abundance proteins for MS, MRM, or immunoassay-based analysis. The nanoparticles are comprised of a shell with defined porosity surrounding a core containing an affinity bait. Harvesting nanoparticle technology $[5,6]$ has been documented to increase the sensitivity of 
mass spectrometry and clinical immunoassay detection by more than 100-fold, with high precision, without increasing the background signal [6]. The added benefit of harvesting nanoparticles is their ability to completely protect the captured analyte from degradation $[5,6]$.

Affinity capture nanoparticles increase the sensitivity of MS by concentrating all the analyte molecules in a large sample volume into a very small volume for introduction into the MS. Nanoparticles also exclude the vast excess of high-abundance proteins, such as albumin, in the original biologic fluid by performing size exclusion. An alternative approach to analyte concentration and pre-separation is antibody affinity capture [7]. In this technology, immobilized antibodies, even those of low specificity for the target analyte, are employed to capture and concentrate the analyte prior to introduction into the MS. Recently, the sensitivity of immuno-MS has reached the $0.5 \mathrm{ng} / \mathrm{ml}$ range when applied to cardiovascular markers [7]. The success of these two different classes of affinity enrichment technologies provides optimism that the sensitivity and pre-analytical variability of MS can be substantially improved. Unfortunately, MS, even with technical enhancements, cannot currently compete with clinical immunoassays on cost, sensitivity, or precision. Instead, MS should play to its strengths: the high accuracy identification of disease-related posttranslational modifications, specific fragments, and protein isoforms. Modified forms of protein are not easily detected by conventional immunoassays. Consequently, for this class of modified analytes, MS would not have much competition.

\section{References}

1. Brown P, Palmer C. The preclinical natural history of serous ovarian cancer: defining the target for early detection. PLoSMed. 2009;6(7):e1000114.

2. Lutz A, Willmann J, Cochran F, Ray P, Gambhir. Cancer screening: a mathematical model relating secreted blood biomarker levels to tumor sizes. PLoSMed. 2008;5(8):e170.

3. Pan S, Aebersold R, Chen R, Rush J, Goodlett DR, McIntosh MW, et al. Mass spectrometry based targeted protein quantification: methods and applications. J Proteome Res. 2009;8(2):787-97.

4. Service RF. Proteomics. Proteomics ponders prime time. Science. 2008;321(5897):1758-61.

5. Longo C, Patanarut A, George T, Bishop B, Zhou W, Fredolini C, et al. Core-shell hydrogel particles harvest, concentrate and preserve labile low abundance biomarkers. PLoS One. 2009;4(3): e4763. Epub 2009 Mar 10.

6. Luchini A, Geho DH, Bishop B, Tran D, Xia C, Dufour RL, et al. Smart hydrogel particles: biomarker harvesting: one-step affinity purification, size exclusion, and protection against degradation. Nano Lett. 2008;8(1):350-61.

7. Gerszten RE, Carr SA. Sabatine integration of proteomic-based tools for improved biomarkers of myocardial injury. Clin Chem. 2010;56(2):194-201. Epub 2009 Dec 18. 